\title{
Antibody Response of Autogenous Splenic Tissue Implanted in the Abdominal Cavity of Mice
}

\author{
Sérgio I. Nunes, MD, PhD, ${ }^{1}$ Alice B. Rezende, MD, ${ }^{1}$ Francisco M. Teixeira, MS, ${ }^{1}$ \\ Ana Paula Ferreira, PhD, ${ }^{1}$ Márcio M.J. Alves, MD, PhD, ${ }^{2}$ Nelson Jamel, MD, PhD, ${ }^{3}$ \\ Raimunda V.C. Assis, MD, PhD, ${ }^{4}$ Henrique C. Teixeira, PhD ${ }^{1}$ \\ ${ }^{1}$ Department of Parasitology, Microbiology and Immunology, Biological Sciences Institute, Federal University of Juiz de Fora, \\ 36036-900 Juiz de Fora, MG, Brazil \\ ${ }^{2}$ Department of Collective Health, Faculty of Medicine, Federal University of Juiz de Fora, 36036-900 Juiz de Fora, MG, \\ Brazil \\ ${ }^{3}$ Department of Surgery, Faculty of Medicine, Federal University of Rio de Janeiro, 21941-590 Rio de Janeiro, RJ, \\ Brazil \\ ${ }^{4}$ Department of Pathology, Faculty of Medicine, Federal University of Juiz de Fora, 36036-900 Juiz de Fora, \\ MG, Brazil
}

\begin{abstract}
There is still controversy about the immunologic function of autotransplanted splenic tissue. In this study, splenic autotransplantation was performed in the abdominal cavity of mice, and the plaqueforming cell (PFC) assay was used to investigate the frequency of antibody-forming cells in response to sheep red blood cell (SRBC) immunization. BALB/c mice were divided into four groups according to the location of the autogenous graft: intraomental (IO), free peritoneal splenosis (FPS), retroperitoneal (RP), and nongrafted control (CT). Thirty days after surgery the mice were immunized intraperitoneally with SRBCs, and 4 days later splenic immunoglobulin $M$ antiSRBC-secreting cells were determined by counting the number of PFCs. All the immunized mice showed increased numbers of PFCs that were about 2 logs higher than those in the the nonimmunized controls $(P<0.005)$. The frequencies of anti-SRBC-producing cells in the tissues grafted in various sites of the abdominal cavity (IO, FPS, RP), in the normal spleen from nonoperated controls (CT), or in the sham-operated control group (SCT) were not notably different ( $5582 \pm 2475$ PFC $/ 10^{7}$ cells for IO; $4849 \pm 1856$ for FPS; $6604 \pm 2903$ for RP; $5940 \pm 5029$ for CT; and $6172 \pm 2203$ for SCT). Similar histology with small architectural variations was observed in all implants; less white pulp was involved, and there was more congestion in the red pulp, with extensive sinusoids and reticular fiber proliferation. This study shows that the $T$ cell-dependent antibody response in implanted splenic tissues is as efficient as in the intact spleen, with no difference between the graft sites studied. This immune response does not depend on the slight architectural variations observed in the splenic implants.
\end{abstract}

$\mathrm{T}$ he importance of the splenic autotransplant technique is justified by the high incidence of total

Correspondence to: Henrique C. Teixeira, $\mathrm{PhD}$, Department of Parasitology, Microbiology and Immunology, Biological Sciences Institute, Federal University of Juiz de Fora, 36036-900 Juiz de Fora, MG, Brazil, e-mail: henrique.teixeira@ufjf.edu.br splenectomy, the spleen being the most affected organ during blunt trauma as well as highly susceptible to iatrogenic incidents. ${ }^{1,2}$ Since the nineteenth century splenectomy has been the treatment advised after splenic trauma, although current studies indicate that this procedure is not exempt from complications. ${ }^{3}$ It is accepted 
that important alterations in hematologic physiology occur after splenectomy, compromising the immune system. The overwhelming postsplenectomy infection syndrome is much more frequent in patients subjected to splenectomy than in the general population, affecting children primarily. ${ }^{4,5}$ Moreover, partial or complete maintenance of the spleen preserves its important capacity for filtration of cells and particles and can avoid alterations of the lipid metabolism that occur after splenectomy. ${ }^{6,7}$ There are still situations where splenectomy is a therapeutic option, as for portal hypertension, but loss of splenic function is not desirable. ${ }^{7,8}$ Methods for conserving the spleen have been described, such as splenorrhaphy, partial splenectomy, subtotal splenectomy, arterial ligatures, and application of hemostatic agents. ${ }^{2,8}$ In situations where complete splenectomy is inevitable, autotransplant is an alternative for preserving a satisfactory immunologic response. $^{9}$

Autogenous grafting of the spleen has been shown to be a viable procedure. Scintigraphic studies have revealed the presence of captive tissue in autotransplanted fragments, ${ }^{1,10,11}$ and histopathologic analysis has shown that the architecture of the autograft is similar to that of normal spleen. ${ }^{12-18}$ In addition, preservation of its phagocytic capacity and the absence of Howell-Jolly corpuscles in peripheral blood have been described after autotransplantation of the spleen. ${ }^{1,7,14}$ Both intraperitoneal and extraperitoneal locations have been used as implant sites for the experimental technique of autogenous spleen graft. $3,18,19$ The most frequent scenario of autotransplantation in humans involves implanting sections of splenic parenchyma into pouches created in the greater omentum because of the favorable revascularization, portal drainage, and omental bacterial clearance. ${ }^{12,17}$ However, there have been complications associated with intraomental splenic autotransplantation, such as torsion of the implant, anemia, and subphrenic abscesses due to necrosis of the implanted tissue. ${ }^{2,19}$ Although many studies have attempted to elucidate the immunologic function of autotransplanted splenic tissue, a general consensus has not been achieved.

To learn more about the immunologic properties of splenic implants, the present study evaluated the frequencies of antibody-forming cells against sheep erythrocytes in immunized BALB/C mice 30 days after splenic autotransplantation in the peritoneal cavity. Grafts were located in the greater omentum and retroperitoneum and as free splenosis in the peritoneal cavity. The histologic examination of these splenic implants is also outlined.

\section{MATERIALS AND METHODS}

Experiments were performed with 80 isogenic mice of the BALB/c lineage, all females, 10 to 12 weeks old. The animals were grouped in the following way: (1) control group (CT), with the mice not subjected to splenectomy; (2) intraomental group (IO), with autotransplantation in the greater omentum; (3) free peritoneal splenosis group (FPS), with autotransplantation in the peritoneal cavity; and (4) retroperitoneal group $(\mathrm{RP})$, with autotransplantation in the retroperitoneum. An additional group of mice was used to study the effect of laparotomy on anti-sheep red blood cell (SRBC) plaque-forming cell (PFC) responses, which we call the sham surgery control (SCT) group. The project was approved by the Committee for Ethics in Animal Experimentation of the Federal University of Juiz de Fora (No. 14/2003).

After anesthesia with phosphate-buffered saline (PBS)chloral hydrate $(4 \%, 8 \mu \mathrm{l} / \mathrm{g}$ body weight, injected intraperitoneally), a midline laparotomy was performed with subsequent splenectomy and binding of the vascular pedicle and short vessels with 5.0 catgut (Shalon, Goiânia, Brazil). All operations were done under sterile conditions. The spleen was cut into six slices about $2.5 \mathrm{~mm}$ thick and kept in physiologic saline at room temperature. In the RP group, the retroperitoneum was opened near the left kidney, and two pieces were placed close to the large abdominal blood vessel without fixation. In the IO group, two fragments were placed in pockets of the greater omentum, where they were sutured with 5.0 catgut. In the FPS group, two fragments were placed loose in the peritoneal cavity without fixation (splenosis). Skin closure was done using a 4.0 running polyglactin suture. In the SCT group, midline laparotomy with subsequent laparorrhaphy was performed.

A direct hemolytic plaque assay was used for estimating the number of immunoglobulin $M$ (IgM)-producing $B$ cells. $^{20,21}$ Seven mice of each group were immunized intraperitoneally with $0.1 \mathrm{ml}$ of a $10 \%$ SRBC suspension (in PBS, pH 7.2) 30 days after splenic autotransplantation. Four days later the animals were sacrificed, and the spleen or splenic autotransplants were removed. Tests were performed in duplicate in solutions containing washed spleen cells or transplant cells in RPMI medium $\left(10^{5}\right.$ to $5 \times 10^{6}$ cells, $\left.0.1 \mathrm{ml}\right)$, washed SRBCs $(1: 3,20 \mu \mathrm{l})$, fresh guinea pig serum as a complement source (1:10, $50 \mu \mathrm{l})$, and $0.5 \%$ Difco agar/DEAE-dextran $(250 \mu \mathrm{l})$. The mixture was poured into a petri dish to form a thin, semisolid top layer. The number of PFCs was determined after 4 hours of incubation at $37^{\circ} \mathrm{C}$ and expressed as PFCs $/ 10^{7}$ cells. 

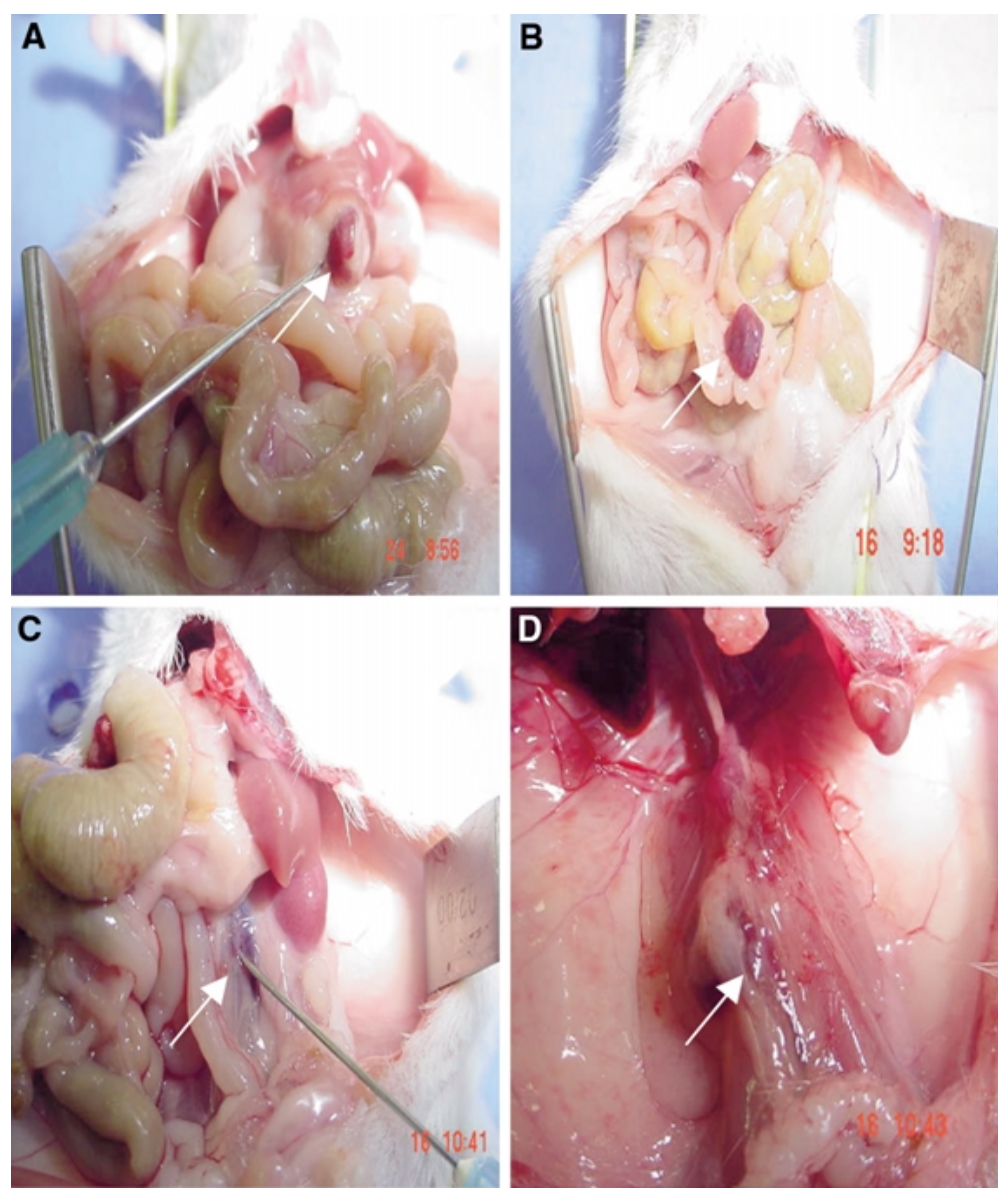

Figure 1. Photograph of splenic implants. Splenic fragments implanted in the greater omentum (A), free peritoneal splenosis (B), and retroperitoneal sites (C, D). Mice were killed by cervical displacement. Arrow: implanted tissue.
For PFC studies from lymph nodes, single cell suspensions from inguinal and periaortic lymph nodes were prepared from nonimmunized or immunized mice killed on day 4 after SRBC immunization. Cell viability was determined by the trypan blue exclusion test. The peripheral blood was obtained from the left axillary artery. Peripheral blood mononuclear cells (PBMCs) were isolated from a heparinized pool (2-3 ml/2 animals) with Histopaque-1077 (Sigma, St. Louis, MO, USA) by density gradient centrifugation at $1700 \mathrm{rpm}$ for 30 minutes at room temperature. PBMCs and lymph node cells were washed twice in PBS and resuspended in RPMI 1640 medium.

For histologic evaluation, the splenic fragments were fixed in 10\% formaldehyde before processing for 14 hours and then embedded in paraffin. Histologic sections about $3 \mu \mathrm{m}$ thick were removed from all the blocks and dyed with hematoxylin-eosin; a histochemical reaction was also undertaken for reticular fibers using ammonia silver solution. All material was evaluated by the same observer who considered: (1) general structures of the organ and the presence of the capsule; (2) the morphologic pattern of the white pulp, the existence of primary and secondary follicles, lymphocyte expansion through reactivity with tingible-body macrophages, and mitoses; (3) the condition of the red pulp and sinusoids, evaluated mainly through the reticulum and determining the diameter; and (4) the presence of giant cells and epithelioid cells.

The groups tested were compared by nonparametric analysis using the Kruskal-Wallis test. A value of $P<0.05$ was considered significant.

\section{RESULTS}

\section{Autogenous Splenic Implants}

Several anatomic sites have been described as suitable for spleen autotransplant, including the omentum and the peritoneum (free peritoneal splenosis). ${ }^{3,12}$ In this study autogenous spleen grafts were prepared in the retroperitoneum and the results compared with those at sites more commonly used (IO and FPS).

All animals that had undergone splenic autotransplantation had gross evidence of viable splenic implants 

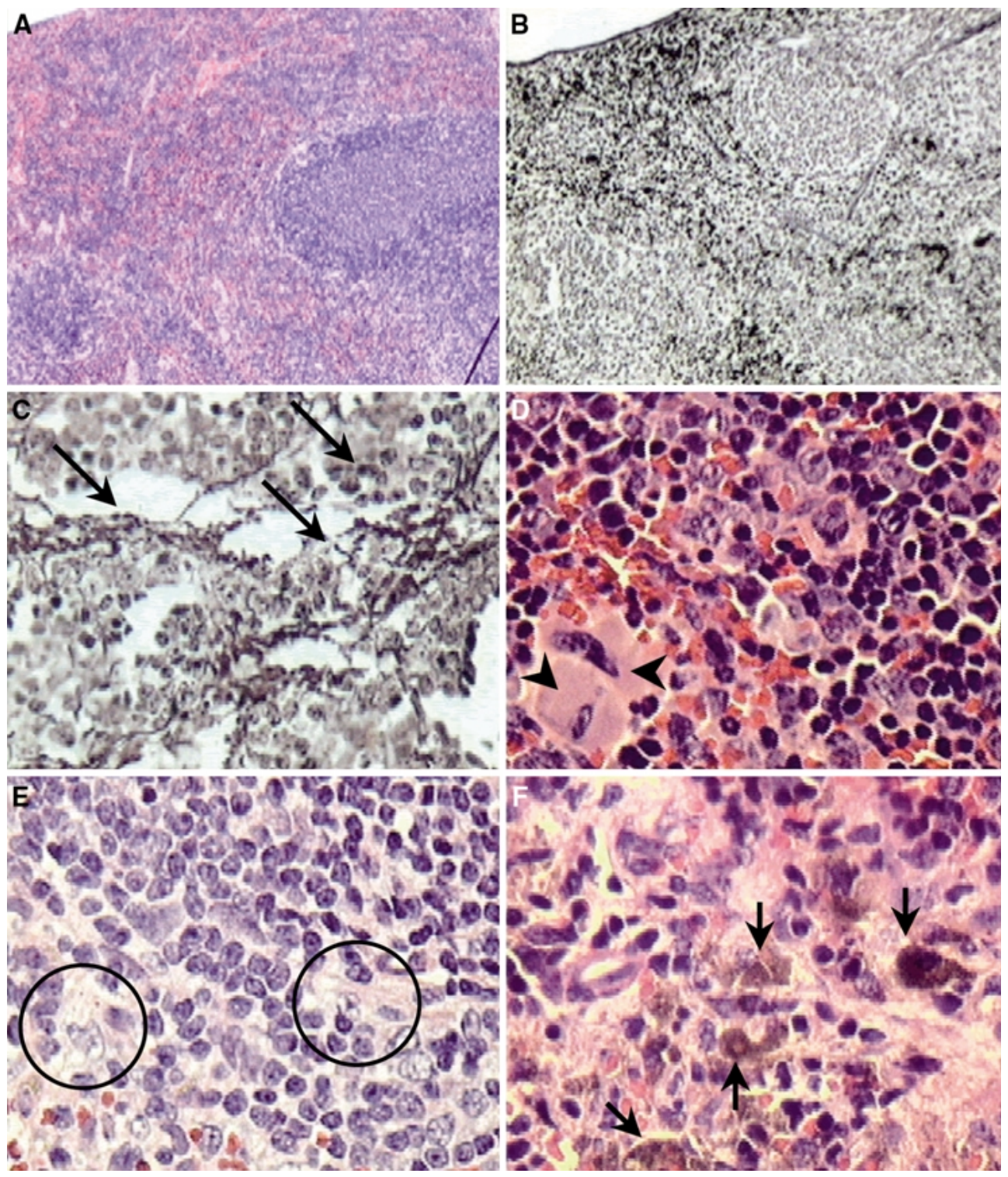

Figure 2. Photomicrograph of a retroperitoneal splenic implant. A. White and red pulp stained with hematoxylin-eosin. B, C. Reticulin fibers (B) and sinusoids (C) with proliferation of the reticulin fibers (long arrows) stained with ammonia silver solution. D-F. Note the presence of giant cells (arrowhead), immunoblasts (circle), and macrophages phagocytosing hemosiderotic pigments (short arrows) in the periphery of secondary follicles.

(A, B $\times 40 ; \mathbf{C} \times 200 ; \mathbf{D}-\mathbf{F} \times 400$ )

(Fig. 1). The survival rate of the transplanted mice was $97.72 \%(43 / 44)$. The average operating times were similar for the groups: $7.3 \pm 2.1$ minutes for $10,8.6 \pm 1.5$ minutes for FPS, and $7.7 \pm 2.0$ minutes for RP. Adhesions were found in $66.6 \%(8 / 12)$ of the 10 animals, $40.0 \%(4 / 10)$ of the FPS animals, and $18.2 \%(2 / 11)$ of the $\mathrm{RP}$ animals.

\section{Histologic Evaluation}

The histologic pattern of control spleens and transplanted splenic fragments were evaluated 34 days after autotransplantation. The main alterations found among the original and transplanted spleens were in respect to loss of the original capsule and formation of thick fibrous tissue on cut surfaces of the fragments as well as the presence of granulation tissue, giant cells, and macrophages phagocytosing hemosiderotic pigments in the periphery (Fig. 2). Animals with implants showed autotransplanted tissue with a structure similar to that of the normal spleen, but minor differences were observed in all implanted groups, such as slight volume reduction of white pulp, congested red pulp, and sinusoids with a more extensive but less organized pattern and with proliferation of the reticular fibers (Fig. 2). No significant histologic differences were observed between the original spleen and implanted splenic fragments from mice stimulated with SRBCs. Epithelioid cells, some giant cells, mitosis, immunoblasts, and macrophages containing cellular remnants were observed in the periphery of secondary follicles in SRBCimmunized mice (Fig. 2). 


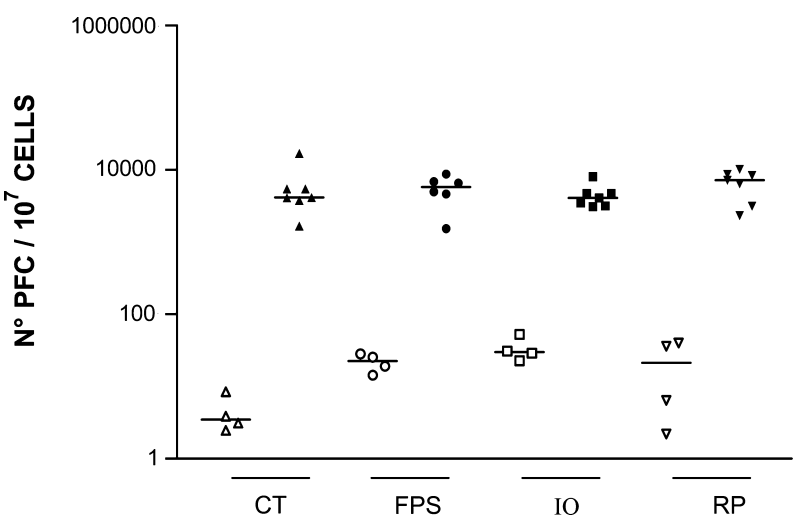

Figure 3. Immunoglobulin M (IgM) plaque-forming cells (PFC) response in splenic implants. Mice were immunized with $2 \times 10^{8}$ sheep red blood cells (SRBC) 30 days after splenic autotransplantation. The number of spleen cells producing anti-SRBC antibodies was determined in the immunized (black symbols) and nonimmunized (white symbols) groups 4 days after immunization by counting the number of PFCs after 4 hours of spleen cell culture. CT: control spleen; FPS: free peritoneal splenosis implant; IO: intraomental implant; RP: retroperitoneal implant. Bars indicate median values.

\section{Plaque-forming Cell Assay}

The hemolytic plaque assay was used to determine if the antibody response of the autotransplanted tissue is preserved; this response is due to the activity of the splenic cells present in the autotransplanted tissue. Figure 3 shows that SRBC-immunized animals had an increase in the number of splenic anti-SRBC PFCs of $\log 2$ in relation to the nonimmunized animals $(P<$ 0.0005). At day 4 after SRBC immunization, the number of anti-SRBC PFCs per $10^{7}$ splenic cells were similar in all of the studied groups $(P=0.582): 5582 \pm 2475$ in the IO group, $4849 \pm 1856$ in the FPS group, $6604 \pm 2903$ in the RP group, and $5940 \pm 5029$ in the control group. To discard the possibility of any effect of laparotomy on the PFC responses, a sham surgery group was evaluated. Anti-SRBC PFC responses were evaluated 4 days after immunization in three groups (34 days after surgery in the groups that underwent operation): the sham surgery group controls (SCT), the splenectomized/ autotransplanted group (AT), and the nonoperated control group (CT). As expected, the number of IgM-PFCs was similar in the three groups studied: $6172 \pm 2203$ in the SCT group, $5305 \pm 585$ in the AT group, and $4237 \pm 903$ in the CT group. The number of PFC responses in the nonimmunized animals was also similar among these groups, at less than $100 \mathrm{PFCs} / 10^{7}$ cells (data not shown).

An additional experiment examining IgM anti-SRBC responses in lymph nodes and peripheral blood was

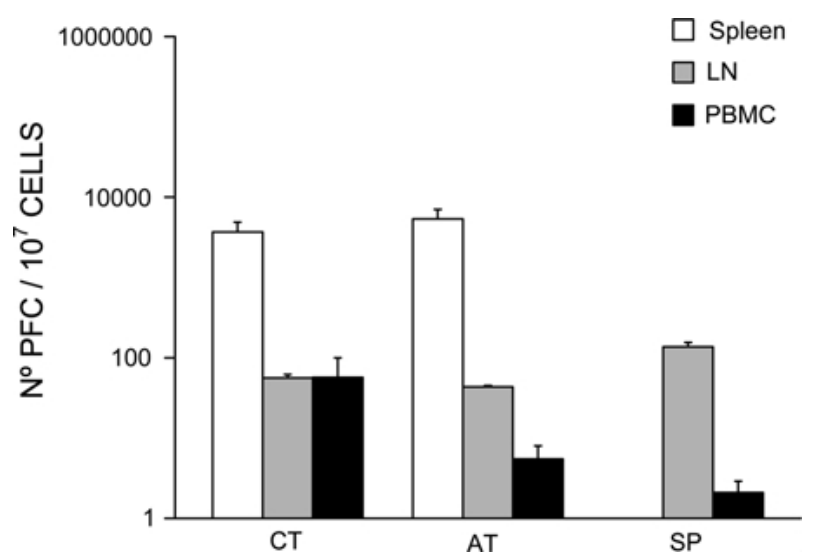

Figure 4. The IgM-plaque forming cells' (PFCs) response in lymph nodes and peripheral blood. Mice were immunized with 2 $\times 10^{8}$ sheep red blood cells (SRBCs) 30 days after splenic autotransplantation. The number of peripheral blood mononuclear cells (PBMCs), lymph node cells (LNs), and splenic cells producing anti-SRBC antibodies was determined 4 days after immunization by counting the number of PFCs. CT: nonoperated control group; AT: autotransplanted in the retroperitoneum; SP: splenectomized and nontransplanted group.

performed to analyze PFC responses in a splenectomized but not autotransplanted group compared to transplanted animals and a control group. The three groups studied were a splenectomized/autotransplanted in the retroperitoneum group (AT); a splenectomized/nontransplanted control group (SP); and a nonoperated control group (CT). The PFC numbers were much higher in spleens or in splenic autotransplanted fragments than in lymph nodes or peripheral blood. In the three groups studied, the PFC numbers in lymph nodes were not significantly different, although they were slightly higher in the SP group. The PBMC anti-SRBC response was measured as an indicator of response from both spleen and lymph nodes. Both groups in which the spleen was removed had a dramatic decline in PBMC-PFCs, but it was even lower in the SP group (Fig. 4).

\section{DISCUSSION}

The most important findings of this study are the following: (1) The histologic and immunologic patterns at the autotransplanted sites do not differ significantly from each other. (2) Histologic structures observed in the implants are similar to normal splenic tissue 34 days after surgery, or 4 days after immunization with SRBCs. (3) The frequency of cells secreting anti-SRBC antibodies in transplanted tissues was comparable to that found in the original organ. 
Splenic autotransplantation is technically easy. There is strong evidence that the autotransplanted graft is anatomically viable, and its histology is similar to that of the normal spleen. ${ }^{12-18}$ The small histologic alterations seen in our study, such as size reduction of the white pulp, increased congestion in red pulp with extensive sinusoids, and reticular fiber proliferation, might be related to the time elapsed between surgery and evaluation (34 days), which is too short a time to detect complete regeneration of the transplanted fragments. However, similar changes in the immunoarchitecture of splenic implants have been found to occur between 41 and 180 days in Lewis rats, suggesting that the term "mature transplants" is a misconception. ${ }^{19,22,23}$ On the other hand, Leemans et al., demonstrated the presence of regenerated splenic tissue 18 weeks after intraomental autotransplantation of splenic tissue in Wistar rats, with a largely restored white pulp. ${ }^{24}$ These discrepancies may reflect strain- and age-related differences. Nevertheless, it is assumed that the younger the recipient and the donor, the better is regeneration and perfusion of the regenerated splenic tissue. ${ }^{24,25}$

Evidence exists that alterations of immunologic markers that naturally occur after splenectomy do not appear after autotransplantation. ${ }^{1,9,26}$ Close to normal serum levels of tuftsin, properdin, IgG, and IgM have been observed after autotransplantation, ${ }^{1,7,14}$, as well as preservation of the phagocytic capacity against pneumococci. This is because opsonin antibodies levels are not altered $^{26,27}$. However, broad conclusions based on various microorganism clearance or host mortality studies published to date on autotransplantation are problematic because of the variety of animal models, infecting organisms, infecting routes, age differences of the animals, and heterogeneous transplantation techniques ${ }^{3}$. The present study showed that all of the autotransplanted splenic grafts studied were immunologically active. Four weeks after transplantation, the number of cells capable of setting up an immune response against SRBCs is the same as that of the original spleen. Fast adaptation and functionality of the transplanted tissue can be decisive factors in guaranteeing the animal with the transplant appropriate resistance to infection.

Our data are in accord with studies in which normal numbers of IgM PFCs were detected 3 to 4 months after splenic implantation in both omental and subcutaneous transplants in rats $^{19}$. Consistent with this assumption, a restored serum antibody response to intravenous challenge with SRBCs was described after intraperitoneal and subcutaneous splenic autotransplantation, with no rise in the asplenic animals ${ }^{27}$. In this study, PFC numbers in lymph nodes were not significantly different among the groups studied after intraperitoneal immunization with SRBCs, although they were slightly higher in the splenectomized group, which may indicate compensation for the lost spleen. Moreover, a dramatic decline in PBMC-PFC numbers was observed in asplenic animals, but it was even lower in the splenectomized but not autotransplanted group. This result suggests that there is a benefit from autotransplantation with respect to PFC responses after splenectomy.

Most important is the study of how these groups respond to infectious agents. Preliminary studies from our laboratory suggest that the capacity of SP mice to control infection caused by intracellular (Mycobacterium bovis) and extracellular (Staphylococcus aureus) infectious agents is markedly decreased in comparison to that in the AT and CT groups (F.M. Teixeira et al., manuscript in preparation). The discrete architectural alterations observed in the splenic implants do not interfere with the $T$ cell-dependent antibody response of the autotransplanted tissue. This is the first study that has measured the number of antibody-secreting cells in splenic implants in mice and the first to use the PFC method to describe the immunologic function of implants in the retroperitoneum.

Several studies have tried to define the site of graft implants, the consensus today being that the abdomen-more precisely the greater omentum-is the best place, mainly owing to its venous drainage being entirely through the portal vein ${ }^{3,28}$. In our study, all the evaluated sites had results similar to those exhibited by normal spleen in relation to the regeneration of splenic tissue. However, there was more formation of adhesions in the tissue implanted in the greater omentum in this study. We cannot formally exclude the possibility that the suture thread used for the intraomental implants contributed to the complications in this scenario. Furthermore, in the case of the omentum autotransplant site, torsion of the omentum has been described as a cause of acute abdomen ${ }^{11,19}$ and aseptic necrosis of the graft ${ }^{29}$. We suspect that the same type of adhesions observed in our model could occur in humans. The subject merits further study.

Our results suggest that the site of autotransplantation does not affect the antibody response. All sites studied showed preservation of tissue architecture and functional ability to respond to $T$ cell-dependent antigens. The autotransplant in the retroperitoneum had the lowest complication rate, and its clinical application in humans deserves attention. 


\section{ACKNOWLEDGMENT}

This research was supported by FAPEMIG (No. 255/ 03) and CNPq (No. 471696/2004-8). S.I.N. was given a doctoral fellowship from CAPES.

\section{REFERENCES}

1. Resende V, Petroianu A. Functions of the splenic remnant after subtotal splenectomy for treatment of severe splenic injuries. Am J Surg 2003;185:311-315.

2. Christo MC, DiDio LJA. Anatomical and surgical aspects of splenic segmentectomies. Ann Anat 1997;179:461-474.

3. Pisters PWT, Pachter HL. Autologous splenic transplantation for splenic trauma. Ann Surg 1994;219:225-235.

4. King $H$, Shumacker HB Jr. Splenic studies. I. Susceptibility to infection after splenectomy performed in infancy. Ann Surg 1952;136:239-242.

5. Horan M, Colebatch JH. Relation between splenectomy and subsequent infection: a clinical study. Arch Dis Child 1962;37:398-412.

6. Jamel MJ, Refineti RA, Silva MM, et al. Papel do remanescente esplênico no perfil lipídico. Arq Bras Cir Dig 2002;15:105-107.

7. Brandt CT, Araujo LB, Barbosa CM. Autotransplantation of spleen tissue in children with mansonic schistosomiasis who underwent splenectomy: evaluation of splenic residual functions. Acta Cir Bras 1998;13:212-216.

8. Buntain WL, Gould HR. Splenic trauma in children and techniques of splenic salvage. World J Surg 1985;9:398-409.

9. Leemans R, Manson W, Snijder JAM, et al. Immune response capacity after human splenic autotransplantation: restoration of response to individual pneumococcal vaccine subtypes. Ann Surg 1999;229:279-285.

10. Ando K, Miyano T, Yamataka A, et al. Torsion of autotransplanted splenic tissue in Gaucher disease. J Pediatr Surg 1997;32:747-749.

11. Sharma R, Mondal A, Kashyap R, et al. Radiolabeled denatured RBC scintigraphy in autologous splenic transplantation. Clin Nucl Med 1996;21:534-536.

12. Mikó I, Bráth E, Furka I, et al. Spleen autotransplantation in mice: a novel experimental model for immunology study. Microsurgery 2001;21:140-142.

13. Gomez L, Egido R, Guarino G, et al. Studio istologico del trapianto splenico sperimentale nei ratti. Minerva Chir 2000;55:741-744

14. Pabst R. Regeneration of autotransplanted splenic fragments: basic immunological and clinical relevance. Clin Exp Immunol 1999;117:423-424.
15. Moore FA, Moore EE, Moore GE, et al. Fivefold enlargement of implants in a splenic autotransplant recipient. Surgery 1993;113:462-465.

16. Petroianu A. Avaliação da função fagocitária em remanescentes de esplenectomia subtotal e auto-implante esplênico autógeno. Rev Bras Hematol Hemoter 2003;25:2530.

17. Weber T, Hanisch E, Baum RP, et al. Late results of heterotopic autotransplantation of splenic tissue into the greater omentum. World J Surg 1998;22:883-889.

18. Cooney DR, Dearth JC, Swanson SE, et al. Relative merits of partial splenectomy, splenic reimplantation and immunization in preventing postsplenectomy infections. Surgery 1979;86:561-569.

19. Liaunigg A, Kastberger C, Leitner W, et al. Regeneration of autotransplanted splenic tissue at different implantation sites. Cell Tissue Res 1992;269:1-11.

20. Jerne NK, Nordin AA. Plaque formation in agar by single antibody-producing cells. Science 1963;140:405.

21. Teixeira HC, Calich LM, Singer-Vermes LM, et al. Experimental paracoccidioidomycosis: early immunosuppression occurs in susceptible mice after infection with pathogenic fungi. Braz J Med Biol Res 1987;20:587-589.

22. Dickerman JD, Horner SR, Coil JA, et al. The protective effect of intraperitoneal splenic autotransplants in mice exposed to an aerosolized suspension of type III Streptococcus pneumoniae. Blood 1979;54:354-358.

23. Pabst $R$, Reilmann $H$. Regeneration of heterotopically transplanted autografts of splenic tissue. Cell Tissue Res 1980;209:137-143

24. Leemans R, Harms G, Rijkers GT, et al. Spleen autotransplantation provides restoration of functional splenic lymphoid compartments and improves the humoral immune response to pneumococcal polysaccharide vaccine. Clin Exp Immunol 1999;117:596-604.

25. Pabst R, Westermann J, Rothkötter HJ. Immunoarchitecture of regenerated splenic and lymph node transplants. Int Rev Cytol 1991;128:215-259.

26. Likhite VV. Protection against fulminant sepsis in splenectomized mice by implantation of autochthonous splenic tissue. Exp Hematol 1978;6:433-439.

27. Schwartz AD, Dadash-Zadeh M, Goldstein R, et al. Antibody response to intravenous immunization following splenic autotransplantation in Sprague-Dawley rats. Blood 1977;49:779-783.

28. Livinstone CD, Levine BA, Sirinek KR. Site of splenic autotransplantation affects protection from sepsis. Am J Surg 1983;146:734-737.

29. Tzoracoleftherakis E, Alivizatos V, Kalfarentzos F, et al. Complications of splenic tissue reimplantation. Ann R Coll Surg Engl 1991;73:83-86. 